\title{
Association of Anti-Phosphatidylcholine Antibodies with Acute Myocardial Infarction: A Comparative Study
}

\author{
1,2 Abdolreza Sotoodeh Jahromi, ${ }^{1}$ Mohammad Shojaei, \\ ${ }^{3}$ Mohammad Reza Farjam and ${ }^{4}$ Abdolhossien Madani \\ ${ }^{1}$ Research Center for Cardiovascular Atherosclerosis, \\ Faculty of Medicine, Jahrom University of Medical Science, Jahrom, Iran \\ ${ }^{2}$ Department of Immunology, Faculty of Medicine, Jahrom University of Medical Science, Jahrom, Iran \\ ${ }^{3}$ Research Center for Social Determinants of Health, \\ Faculty of Medicine, Jahrom University of Medical Science, Jahrom, Iran \\ ${ }^{4}$ Research Center for Social Determinants in Health Promotion, \\ Faculty of Public Health, Hormozgan University of Medical Science, Bandarabbas, Iran
}

Received 2013-10-20, Revised 2013-10-24; Accepted 2013-10-28

\begin{abstract}
Many factors play a role in Acute Myocardial Infarction (AMI). One those anti-Phospholipid (aPL) antibodies, that may act in the induction of immunological response leading to the development of AMI. Anti-Phosphatidylcholines (PC) antibody is detected in various diseases like rheumatoid arthritis, systemic lupus erythematosus and anti-phospholipid antibody syndrome. The study of anti-PC antibody in AMI might shed light on etiologic mechanisms in the pathogenesis of acute coronary syndromes. This study was designed to investigate whether prevalence of anti-PC antibodies, in patients who had AMI and to analyze their relationship with traditional cardiovascular risk factors. The prevalence of anti-PC IgG and IgM in a well characterized group of patients with AMI as a case group and in age and sex matched healthy subjects as control group. Sera from the case and the control groups were tested to evaluate the presence of $\mathrm{IgG}$ and IgM isotypes to anti-PC by ELISA method. The prevalence of anti-PC IgG and also IgM in the case group resulted significantly higher than in the control group with AMI $(\mathrm{p}<0.005)$. Our findings suggest that anti-PC antibodies seemed to play a role in AMI, independent risk factors for AMI, which may represent a link between autoimmunity and atherosclerosis in patients with AMI. Further studies with bigger sample size including patients with AMI and healthy people are needed to explore the exact role of anti-PC antibodies in AMI.
\end{abstract}

Keywords: Anti-Phosphatidylcholines (PC) Antibodies, Acute Myocardial Infarction (AMI), AntiPhospholipid (aPL) Antibodies, Cardiovascular Ischemia

\section{INTRODUCTION}

Antiphospholipid antibodies (aPL) in addition to repeated miscarriages and pregnancy complications (Jahromi et al., 2010a) are associated with cardiovascular diseases (Jahromi et al., 2013; Shojaie and Jahromi, 2011; Jafarzadeh et al., 2011). However their pathogenic mechanisms are still matter of investigation.
In addition to the Classical Lupus Anticoagulant (LAC) and Anti-Cardiolipin Antibodies (ACA), other anti-Phospholipid Antibodies (aPL) were shown to target anionic phospholipids and other plasma proteins, including phosphatidylethanolamine, protein $\mathrm{C}$, protein S, 32-Glycoprotein I (beta2-gpI) and annexin V (Tincani et al., 2010).

Corresponding Author: Mohammad Shojaei, Research Center for Cardiovascular Atherosclerosis, Faculty of Medicine, Jahrom University of Medical Science, Jahrom, Iran 
Myocardial Infarction (MI) is the combined result of environmental factors and personal predispositions. Factors such as low serum adiponectin (Shojaie et al., 2009a) and low annexin V levels (Shojaie et al., 2009b) and infectious diseases such as Mycoplasma pneumonia (Pourahmad et al., 2009) are a part of involving factors in AMI. It has been demonstrated that the immunopathological such as anti-beta2 glycoprotein-I antibody (Shojaie and Jahromi, 2011) and Inflammatory processes (Jahromi et al., 2010a) play important roles in the initiation and development of AMI.

The results of some studies also demonstrated an association between anti-PL antibody and IHD (Shojaie and Jahromi, 2011; Jafarzadeh et al., 2011). It should be noted that genetic factors as well as other traditional risk factors such as smoking, hypercholesterolemia, diabetes mellitus and hypertension may contribute to IHD development and these parameters differ among various population. Although there are a few studies on the association of some autoantibodies with AMI, more epidemiological data are required to confirm their significance as independent risk factors in cardiovascular diseases. Moreover, the data on the relationship of autoantibodies with traditional risk factors of AMI is scarce. Therefore, this study was conducted to evaluate the serum levels of Anti-Phosphatidylcholines (PC) antibodies (IgG and IgM) in Iranian patients with AMI and also to clarify their association with traditional risk factors of the disease.

\section{MATERIALS AND METHODS}

\subsection{Subjects}

A total of 90 consecutive patients (aged 41-67 years) with AMI including 67 men and 23 women who were admitted to Peymanieh Hospital of Jahrom in southwest of Iran, were enrolled to this crosssectional, case-control study.

AMI was diagnosed by the presence of two of the following criteria: (i) prolonged chest pain compatible with AMI, (ii) typical ECG changes, (iii) rising of cardiac enzymes such as creatine kinase and lactate dehydrogenase. UA was defined according to the Braunwald's classification and all patients had chest pain at rest with definite ischemic electrocardiographic changes such as ST-segment changes and/or T-wave inversion (Antman et al., 2008).

Exclusion criteria were valvular heart disease, surgery, trauma during the prior month, cardiomyopathy, liver disease, renal failure, arthritis, malignant diseases, other inflammatory diseases (such as SLE and RA) and oral anticoagulant therapy. In patients with AMI, the serum concentration of Anti-PC antibodies was measured during 3-5 days after admission.

A age, sex and other CAD risk factors such as Hypertension (HTN), Diabetes Mellitus (DM) and Hyper Lipidemia (HLP) matched group $(\mathrm{n}=90)$ with similar geographic and socioeconomic backgrounds without any ischemic heart disease were used as a control group. All control subjects were basically healthy, with no acute or chronic illnesses and did not use any drugs. The healthy control group was recruited from blood donors attending Jahrom Blood Transfusion Center. Peripheral blood (3 milliliter) was collected from the two groups and the serum was separated and stored at $-20^{\circ} \mathrm{C}$.

The study protocol was approved by research ethics committee of Jahrom University of Medical Sciences and informed consents were obtained from all participants before enrollment.

The patient or his legal representative provided written informed consent. Historical, demographic and clinical data were obtained through a review of medical records and interviews with patients and their families. The risk factors for myocardial infarction were as follows: (1) age, sex; (2) history of hypertension (diagnosis confirmed when the systolic or diastolic pressures were $>160$ or $95 \mathrm{mmHg}$, respectively, or when the patient was using antihypertensive medication) 12 ; (3) smoking, according to the criteria of the British Council for Medical Research; (4) history of heart disease (atrial fibrillation or coronary heart disease, defined as previous myocardial infarction, angina, or revascularization procedure); (5) history of diabetes mellitus, according to the medical history or the use of insulin or an oral antidiabetes drug; (6) hypercholesterolemia, based on total cholesterol $>200 \mathrm{mg} \mathrm{dL}^{-1}$, LDL-cholesterol $>130 \mathrm{mg}$ $\mathrm{dL}^{-1}$, or total cholesterol/HDL-cholesterol ratio $>5$ (Donahue et al., 1988).

Blood samples $(5 \mathrm{cc}$ ) were obtained by venipuncture from the patients immediately after admission before starting any IV medications by skilled personnel. Blood samples were centrifuged and frozen within, at most, $2 \mathrm{~h}$ after collection and stored at $-20^{\circ} \mathrm{C}$ until laboratory testing with ELISA. Serum anti-PC IgG and anti-PC IgM levels was determined by quantitative and qualitative ELISA method respectively, following the manufacturer instructions using IBL company, REF: RE70461.

Statistical analyses were performed by SPSS (version 17; SPSS, Inc., Chicago, IL). Data were expressed as mean \pm SD. Continuous variables with little-to-mild skewness were summarized as mean $\pm \mathrm{SD}$ and compared using Student's t-test. 
Abdolreza Sotoodeh Jahromi et al. / American Journal of Immunology 9 (4): 116-119, 2013

Table 1. Demographic, clinical characteristics and laboratory finding for the case and the control groups

\begin{tabular}{llll}
\hline Variables & Patients group & Control group & P-value \\
\hline Age (year) & $9.63 \pm 62.66$ & $10.63 \pm 60.53$ & 0.609 \\
Male (\%) numbers & $67(74.40 \%)$ & $68(75.60 \%)$ & 0.863 \\
High blood pressure (\%) numbers & $39(43.33 \%)$ & $28(31.10 \%)$ & 0.079 \\
Smoker (\%) numbers & $40(44.40 \%)$ & $32(35.60 \%)$ & 0.287 \\
IDDM * (\%) numbers & $24(26.70 \%)$ & $28(31.10 \%)$ & 0.622 \\
Total cholesterol * Mg/dL & $176.13 \pm 40.30$ & $168.80 \pm 43.20$ & 0.104 \\
HDL-C * Mg/dL & $44.20 \pm 9.11$ & $42.83 \pm 10.29$ & 0.328 \\
LDL-C * Mg/dL & $118.05 \pm 36.32$ & $98.22 \pm 30.64$ & 0.093 \\
TG $*$ Mg/dL & $126.53 \pm 72.18$ & $114.84 \pm 41.52$ & 0.104 \\
FBS * Mg/dL & $134.20 \pm 54.48$ & $127.08 \pm 53.91$ & 0.495 \\
Positive Anti-Phosphatidylcholine IgG & $27(30.00 \%)$ & $10(11.10 \%)$ & 0.002 \\
Positive Anti-Phosphatidylcholine IgM (\%) numbers & $15(16.70 \%)$ & $(3.30 \%) 3$ & 0.003 \\
\hline
\end{tabular}

*IDDM $=$ Insuline Dependent Diabetes Mellitus, NIDDM = Non-Insuline Dependent Diabetes Mellitus, HDL-C = High Density Lipoprotein-Cholesterol, LDL-C = Low Density Lipoprotein-Cholesterol, FBS = Fasting Blood Sugar

\section{RESULTS}

The demographic and clinical characteristics of the study groups, as well as laboratory variables are shown in Table 1.

In the patient group 16 cases $(18.80 \%)$ had Non-St Elevation MI (NSTEMI) and $74(82.20 \%)$ had ST Elevation MI (STEMI).

There was no significant difference between the two groups regarding the following variables: age, sex, HTN, DM, LDL, HDL, total cholesterol and TG.

We examined the association between positive anti$\mathrm{PC}$ IgG and IgM tests and selected cardiovascular risk factors. There was a significant association between positive anti-PC IgG and IgM tests with Type hypertension. But there was not found any significant association between positive anti-PC $\operatorname{IgG}$ and $\operatorname{IgM}$ test with Type 1 DM, Type 2 DM, age, sex, LDL, HDL, TG, total cholesterol and adjusted smoking.

There was not found significant difference between positive anti-PC IgG tests in patients with STEMI and those with NSTEMI $(73.30 \%$ Vs $25.90 \%),(p=0.186)$ and also there was not found significant difference between positive anti-PC IgM tests in patients with STEMI and those with NSTEMI (74.10\% Vs 26.70\%), $(\mathrm{p}=0.324)$.

\section{DISCUSSION}

The results of the present study showed that the prevalence of positive anti-PC IgG and also IgM antibodies in patients with AMI were significantly higher than that observed in healthy control group. These associations were independent of traditional risk factors. We found high prevalence of anti-PC antibodies in patients with AMI Vs healthy control group consistent with current concepts on the immune pathogenesis of atherosclerosis. However, the precise mechanisms of involvement of anti-PC antibodies in the pathogenesis of AMI remain to be determined.

We could not find any research finding about anti-PC antibodies in AMI to compare its results with the results of present study.

Other aPLs have been reported to have association with CAD and AMI (Shojaie and Jahromi, 2011; Jafarzadeh et al., 2011; Greco et al., 2010).

There was not found correlation between anti-PC IgG and also IgM and standard cardiovascular risk factors such as smoking, hypertension and diabetes, as seen about other apl antibodies in a previous study (Shojaie and Jahromi, 2011; Jahromi et al., 2010b).

In this study there was a significant association between anti-PC IgG with HTN in the case and the control groups. The same results were found about anti$\beta 2$ GPI IgG in AMI in other studies (Yamada et al., 2010). These results suggest that anti- $\beta 2$ GPI IgG is independent of these variables.

As there were not significant differences between positive anti-PC antibodies test in patients with STEMI and those with NSTEMI, it can be concluded that antiPC IgG and IgM do not participate in kind of AMI (STEMI vs NSTEMI). But, unfortunately we did not any data indicating association of anti-PC IgG and IgM with these parameters to compare the results.

\section{CONCLUSION}

These results show an association between AMI and high prevalence of positive tests for anti-PC IgG and IgM. The results of this study in parallel with the 
data from other studies (Shojaie and Jahromi, 2011; Jafarzadeh et al., 2011; Greco et al., 2009; Urbanus et al., 2009) support the potential important role of aPL antibodies in AMI.

Further studies with larger sample size of patients and healthy subjects are recommended to explore the precise role of anti-PC IgG and IgM in AMI.

\section{ACKNOWLEDGEMENT}

This study was completely financed by Jahrom University of Medical Sciences. The authors are grateful to the patients and the control subjects for their enrollments in this study.

\section{REFERENCES}

Antman, E.M., M. Hand, P.W. Armstrong, E.R. Bates and L.A. Green et al., 2008. 2007 Focused Update of the ACC/AHA 2004 Guidelines for the Management of Patients With ST-Elevation Myocardial Infarction: A report of the American College of Cardiology/American Heart Association Task Force on Practice Guidelines: Developed in collaboration With the Canadian Cardiovascular Society endorsed by the American Academy of Family Physicians: 2007 Writing Group to Review New Evidence and Update the ACC/AHA 2004 Guidelines for the Management of Patients With STElevation Myocardial Infarction, Writing on Behalf of the 2004 Writing Committee. Circulation, 15: 296-329. DOI: 10.1161/CIRCULATIONAHA.107.188209

Donahue, R.P., R.D. Abbott, D.M. Reed and K. Yano, 1988. Physical activity and coronary heart disease in middle-aged and elderly men: The honolulu heart program. Am. J. Publ. Health, 78: 683-685.

Greco, T.P., A.M. Conti-Kelly, J.R. Anthony, T. Greco and R. Doyle et al., 2010. Oxidized-LDL/beta(2)glycoprotein I complexes are associated with disease severity and increased risk for adverse outcomes in patients with acute coronary syndromes. Am. J. Clin. Pathol., 133: 737-743. PMID: 20395520

Greco, T.P., A.M. Conti-Kelly, J.R. Greco, R. Doyle and E. Matsuura et al., 2009. Newer antiphospholipid antibodies predict adverse outcomes in patients with acute coronary syndrome. Am. J. Clin. Pathol., 132: 613-620. PMID: 19762540

Jafarzadeh, A., M. Poorgholami, M. Nemati and M.T. Rezayati, 2011. High serum levels of rheumatoid factor and anti-phosphatidylserine antibody in patients with ischemic heart disease. Iran J. Immunol., 8: 34-44. PMID: 21427494
Jahromi, A.S., M. Shojaei, M.R. Farjam and A. Madani, 2013. The presence of antiphosphatidylethanolamine antibodies in acute myocardial infarction. OnLine J. Biol. Sci., 13: 126130. DOI: $10.3844 /$ ojbsci.2013.126.130

Jahromi, A.S., M. Shojaie and A. Madani, 2010a. Cardiotrophin-1 in patients with acute myocardial infarction. Am. J. Applied Sci., 7: 1190-1194. DOI: 10.3844/ajassp.2010.1190.1194

Jahromi, A.S., M. Shojaie, S. Dana and A. Madani, 2010b. Anti-cardiolipin antibody in acute myocardial infarction. Am. J. Immunol., 6: 11-14. DOI: 10.3844/ajisp.2010.11.14

Pourahmad, M., S.A. Jahromy and M. Shojaei, 2009. Association of mycoplasma pneumonia infection with myocardial infarction. Am. J. Immunol., 5: 84-88. DOI: 10.3844/ajisp.2009.84.88

Shojaie, M. and A.S. Jahromi, 2011. Anti- $\beta 2-$ glycoprotein-i antibody in acute myocardial infarction. Am. J. Applied Sci., 8: 758-761. DOI: 10.3844/ajassp.2011.758.761

Shojaie, M., A. Sotoodah and G. Shafaie, 2009a. Is adiponectin associated with acute myocardial infarction in Iranian non obese patients. Lipids Health Dis., 8: 17-17. DOI: 10.1186/1476-511X-817

Shojaie, M., A. Sotoodah, S. Roozmeh, E. Kholoosi and S. Dana, 2009b. Annexin V and anti-annexin V antibodies: Two interesting aspects in acute myocardial infarction. Thromb. J., 21: 7-13. DOI: 10.1186/1477-9560-7-13

Tincani, A., C. Casu, S. Cartella, T. Ziglioli and R. Cattaneo, 2010. Antiphospholipid antibody: Laboratory, pathogenesis and clinical manifestations. Reumatismo, 62: 65-75. PMID: 20390120

Urbanus, R.T., B. Siegerink, M. Roest, F.R. Rosendaal and P.G. de Groot et al., 2009. Antiphospholipid antibodies and risk of myocardial infarction and ischaemic stroke in young women in the RATIO study: A case-control study. Lancet Neurol., 8: 9981005. PMID: 19783216

Yamada, H., T. Atsumi, O. Amengual, T. Koike and I. Furuta et al., 2010. Anti-beta2 glycoprotein-I antibody increases the risk of pregnancy-induced hypertension: A case-controlled study. J. Reprod. Immunol., 84: 95-99. PMID: 20004024 